\title{
Modeling discontinuous potential distributions using the finite volume method, and application to liquid metal batteries
}

\author{
Norbert Weber ${ }^{\mathrm{a}, \mathrm{b}}$, Steffen Landgraf ${ }^{\mathrm{a}}$, Kashif Mushtaq ${ }^{\mathrm{b}, \mathrm{c}, \mathrm{d}}$, Michael Nimtz $^{\mathrm{a}}$, \\ Paolo Personnettaz ${ }^{\mathrm{a}}$, Tom Weier ${ }^{\mathrm{a}}$, Ji Zhao ${ }^{\mathrm{b}}$, Donald Sadoway ${ }^{\mathrm{b}}$ \\ ${ }^{a}$ Helmholtz-Zentrum Dresden - Rossendorf, Bautzner Landstr. 400, 01328 Dresden, \\ Germany \\ ${ }^{b}$ Department of Materials Science and Engineering, Massachusetts Institute of Technology, \\ 77 Massachusetts Avenue, Cambridge, MA 02139-4307, United States \\ ${ }^{c}$ LEPABE, Department of Chemical Engineering, Faculty of Engineering, University of \\ Porto, Portugal \\ ${ }^{d}$ Department of Mechanical Engineering, School of Mechanical and Manufacturing \\ Engineering, National University of Sciences and Technology, Islamabad, Pakistan
}

\begin{abstract}
The electrical potential in a battery jumps at each electrode-electrolyte interface. We present a model for computing three-dimensional current and potential distributions, which accounts for such internal voltage jumps. Within the framework of the finite volume method we discretize the Laplace and gradient operators such that they account for internal jump boundary conditions. After implementing a simple battery model in OpenFOAM we validate it using an analytical test case, and show its capabilities by simulating the current distribution and discharge curve of a Li ||Bi liquid metal battery.

Keywords: potential distribution, current distribution, internal boundary, internal jump, OpenFOAM, finite volume method, liquid metal battery
\end{abstract}

\section{Introduction}

The objective of this article is twofold: we want to model both, the current distribution in, and charge-discharge curves of electrochemical cells. For good overviews on the subject, see [1 13 . As a first step, we will discuss only modeling of the time-dependent cell potential.

Preprint submitted to Electrochimica Acta

June 24, 2019 


\subsection{Battery voltage, overpotentials and polarization curve}

Most battery models do not describe the distribution of current and potential. Instead, they provide only the total cell voltage, which can be simply calculated as the difference of the open circuit potential and the sum of all overvoltages 448 . We are here especially interested in the ohmic overpotential. In many batteries, the electrolyte has by far the highest resistance. In such cases the ohmic losses can be calculated analytically or by potential theory, i.e., by solving a Laplace equation in the electrolyte [911. More advanced models are used for porous electrodes, but rely on the same Laplace equation [12 16]. Even if the full potential distribution is computed in the electrolyte, all these models use in the end only a scalar voltage loss to compute the cell potential. They are therefore limited in many ways - and can not account for effects such as the potential drop in poorly conducting electrodes (e.g. Se, S [17]), complicated geometries where much current flows only through a part of the electrolyte, local activation overpotentials, or liquid electrodes where diverging current drives convection [18, 19].

In order to account for all these effects, we need to know the potential distribution in the battery. Fig. 1 shows a one-dimensional example - an example which is strongly simplified. It relies on the macroscopic approach, i.e., we do not resolve the electrochemical double layer, and consider all phases to be electrically neutra ${ }^{1}$ [3, 13, 21]. Within these assumptions, the potential jumps at both electrode-electrolyte interfaces according to the Nernst equation. As illustrated in fig.1 1 the cell voltage is simply the difference of both potential jumps. During operation of the electrochemical cell, the potential jumps are directly reduced by concentration and activation overpotentials. Additionally, the cell potential decreases by the ohmic overpotential, as illustrated in fig. 1 $\mathrm{b}$.

\footnotetext{
${ }^{1}$ For simulating alternating current response of electrochemical cells it will be necessary to consider the effects of the electrochemical double layer. For more information on the subject, see, e.g., 20].
} 


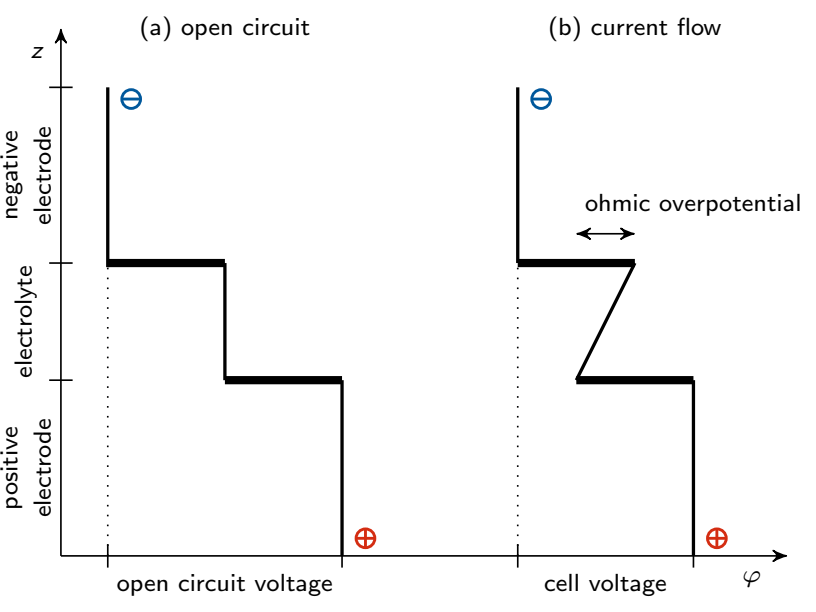

Figure 1: Schematic voltage profile in an electrochemical cell with open (a) and closed (b) circuit at discharge. For an excellent discussion of such profiles, see [4 22, 24].

\subsection{Modeling current and potential distributions}

After describing one-dimensional potential profiles in the previous section, we will proceed with a discussion of 3-dimensional modeling. Within continuum mechanics, potential distributions can be obtained by solving a Laplace equation

$$
\nabla \cdot \sigma \nabla \varphi=0
$$

where $\varphi$ denotes the electric potential and $\sigma$ the ionic or electronic conductivity. This simple approach is only fully correct if there are no concentration gradients in the electrolyte [6, 25] and if double layer charging is neglected [3. The challenge of solving equation 1 is the potential jump between electrolyte and electrodes. Generally, two solutions exist: the single- and the multi-potential approaches [26]. The latter relies on the idea of defining separate ionic and electronic potentials [27, 28, i.e., several Laplace equations are solved. The offset, i.e., the jump between the potentials, is typically defined by a volumetric source term in an envelope layer near the interface [29]. Such a model is ideally suited for porous electrodes, such as the catalyst layer in a fuel cell. The porous electrode and electrolyte are treated as superimposed continua [12, 27] of 
finite thickness [6]; a charge leaving the solid matrix must enter the pore liquid [30. The superficial charge transfer - described by Faraday's law - needs to be transferred into only a volumetric source term [12, 21]. Such multi-potential porous electrode models are extremely popular - for more details, see especially [12, 13, 30 32. Please note that many researchers exclude the potential jump due to the Nernst potential, but model only the jump due to the activation overpotential.

Compared to the above described porous model, the single-potential approach is less common. It uses only one single potential field for the whole cell. The potential jump is not implemented as a source term, but as an internal jump boundary condition. Such models are also known as "interface models" because they assume the interface to be infinitely thin [3]. We will implement such a model and describe in the following how the equations are set up and how they are solved.

\subsection{Solving coupled equation systems with internal jumps}

As described in the previous section, the electric potential between electrodes, electrolyte and conductors needs to be coupled. We will denote this by the term "region coupling". The voltage of electrochemical cells depends, among others, on temperature and concentration. Each of these fields is described by its own equation - and all of them are coupled. We will denote that as "equation coupling". Newman's original method for coupling different equations [33] is based on block-matrices, i.e., all equations are discretized in one matrix and solved in one step 34 36. This method was slightly extended [37 40] and used for many different applications. For a good overview about the method, see [41]. An alternative to block-coupling is a segregated solver: it solves each equation separately. As the equations are coupled, this process must be repeated iteratively until convergence is reached. On the one hand, the block-coupled single-matrix approach is surely better suited for highly coupled equations, and maybe for strongly non-linear equations [42]. On the other hand, the segregated approach is easier to implement and needs much less computer 
memory. Both approaches have advantages and drawbacks - there is not a single, and perfect solution for equation coupling.

Similarly, region coupling can be obtained by solving the potential on one global mesh, or by solving a separate equation for each conductor. The latter is well known in OpenFOAM. Using appropriate boundary conditions, a potential jump at the interface can easily be implemented [43, 44]. However, a relatively time consuming iteration between all regions is necessary. This can be extremely slow 45, because the regions are coupled only at the interface (and not in the volume). Solving the potential in all regions in a coupled way in one matrix is definitely the better way. For possible implementations of internal potential boundary conditions, see [6] 48.

Finally, the equation system can be set-up and solved using the finitedifference (FDM), finite-element (FEM), boundary element or finite-volume method (FVM) 2, 9. During the first years, the FDM was surely the most widely used approach for modeling potential problems 6]. Today, commercial codes such as COMSOL [49] (FEM), STAR-CCM+ 50] (FVM) and Ansys Fluent [51] (FVM) also allow for solving potential problems with internal jumps. However, their solution algorithm is not always published.

\subsection{Our approach}

We will model potential distributions with internal jumps using a singlepotential approach. The set of equations will be solved in a segregated manner, while a block-matrix will be used for region coupling of the electric potential. We further use a multi-mesh approach [8, 45]. This means, we solve certain variables (as the potential) on a global mesh, but others (such as concentration) only in the electrodes. The equations are discretized using the finite volume method, and the model is implemented in the open source CFD library OpenFOAM v1806 [52]. 


\section{Model}

We present in this section the most simple model for the potential distribution in a concentration cell. With little effort, it can be extended to arbitrary electrochemical cells. We use the parent child-mesh technique, i.e., we provide one global mesh for the full geometry and a second mesh for the positive electrode only. Where necessary, we map variables between both meshes. The following simplifications apply:

- fluid flow, heat transfer and variation of the layer thickness are neglected

- the charge transfer overpotential is neglected

- the electrochemical double layer is not resolved; we assume discrete potential jumps at the interfaces

- concentration gradients in the electrolyte are neglected [53]

In a first step we solve the concentration $c$ in the positive electrode as

$$
\frac{\partial}{\partial t} c=\nabla \cdot(D \nabla c)
$$

where $D$ denotes the diffusion coefficient. We apply zero flux boundary conditions at all interfaces except the electrode-electrolyte boundary. Here we set the concentration gradient corresponding to the current density $\boldsymbol{J}$ as

$$
\nabla_{n} c=-\frac{\boldsymbol{J}}{z F D} \boldsymbol{n}
$$

where $\nabla_{n}$ denotes the interface-normal gradient, $z$ the number of electrons, $F$ the Faraday constant and $\boldsymbol{n}$ the interface normal vector.

The local concentration at the electrode-electrolyte interface determines the jump in potential. The latter could be computed from the Nernst equation using measured activities. However, for simplicity and to avoid numerical instabilities [42], we directly fit the measured open circuit voltage. We save this potential jump at the corresponding face centers and map it to the parent mesh. Please note that the potential jump is applied only at the interface between electrolyte and the positive electrode (and not at the negative electrode). 
On the global mesh we solve a Laplace equation for the electric potential $\varphi$ as

$$
\nabla \cdot \sigma \nabla \varphi=0
$$

where $\sigma$ denotes the electrical conductivity. Finally, the current density is computed as

$$
\boldsymbol{J}=\sigma \nabla \varphi
$$

and mapped to the electrode-electrolyte interface where it is needed to compute the boundary condition for the concentration. The potential jump at the electrolyte-positive electrode interface is accounted for when discretizing the gradient and Laplace operator as described in the next section.

We discretize the equations using the implicit Euler scheme for time and second order schemes for all spatial terms. The potential is solved using a PCG, and the concentration by a multigrid solver [54].

\section{Discretization of jump condition}

Solving the electrical potential on one single mesh, one needs to account for the jumping potential in two terms. The first is the Laplace equation 4 where the potential jump will appear in form of an additional source term. Secondly, when computing the current density by equation 5 , both the potential jump and the discrete change of conductivity has to be observed.

\subsection{Laplace operator}

Within the finite volume method, the Laplace operator can be discretized using the Gauss theorem as [55]

$$
\nabla \cdot \sigma \nabla \varphi=\sum_{f} \sigma_{f} \boldsymbol{S}(\nabla \varphi)_{f}
$$

with $\boldsymbol{S}$ denoting the face area vector and $(\nabla \varphi)_{f}$ the gradient at the face. The face conductivity $\sigma_{f}$ is consistently discretized from the cell centered values ${ }^{2}$

\footnotetext{
${ }^{2}$ We use the word "cell" in section 3 in the sense of grid cell or control volume.
} 
using harmonic interpolation [45. Denoting the potential in the owner and neighbor cell of face $f$ by $\varphi_{\mathrm{P}}$ and $\varphi_{\mathrm{N}}$ we can also write

$$
\nabla \cdot \sigma \nabla \varphi=\sum_{f} \boldsymbol{S} \sigma_{f}(\nabla \varphi)_{f}=\sum_{f}|\boldsymbol{S}| \sigma_{f} \frac{\varphi_{\mathrm{N}}-\varphi_{\mathrm{P}}+\Delta \varphi}{|\boldsymbol{d}|}
$$

where $\Delta \varphi$ denotes the potential jump at the interface and $\boldsymbol{d}$ the vector connecting both cell centers. Writing the matrix equation as

$$
A_{d}+A_{o}=S
$$

we find the off-diagonal coefficients as

$$
A_{o}=\frac{\sigma_{f}|\boldsymbol{S}|}{|\boldsymbol{d}|}
$$

and the diagonal coefficients as

$$
A_{d}=-\sum_{n} \frac{\sigma_{f}|\boldsymbol{S}|}{|\boldsymbol{d}|}=-\sum_{r} A_{r, o}
$$

where the index $r$ denotes related cells, i.e., cells which share a common face ${ }^{3}$ Finally, the source term is

$$
S=\sum_{f}-\frac{\sigma_{f}|\boldsymbol{S}| \Delta \varphi}{|\boldsymbol{d}|}
$$

The potential jump $\Delta \varphi$ will appear only at the electrode-electrolyte interface; for all grid cells not touching the interface the source term will therefore be zero.

The above described procedure applies perfectly to orthogonal meshes. It can be easily extended to arbitrary polyhedral control volumes using the overrelaxed correction approach [54]58].

\subsection{Gradient operator}

Using the Gauss theorem, the gradient of the electric potential is discretized as 55 .

$$
\nabla \varphi=\frac{1}{V} \sum_{f} \boldsymbol{S} \varphi_{f}
$$

\footnotetext{
${ }^{3}$ For details, see https://openfoamwiki.net/index.php/OpenFOAM_guide/Matrices_in_ OpenFOAM
} 
where $V$ denotes the cell volume and $\varphi_{f}$ the potential at a face. The latter is determined from the potential of the owner $(\mathrm{P})$ and neighbor $(\mathrm{N})$ cell using two continuity conditions. Firstly, we assume the potential itself to be continuous (or jumping) over the face as

$$
\varphi_{\mathrm{wN}}=\varphi_{\mathrm{wP}}+\Delta \varphi
$$

and secondly we ensure continuity of normal currents by

$$
\sigma_{\mathrm{N}} \nabla \varphi_{\mathrm{N}} \cdot \boldsymbol{n}_{\mathrm{N}}=\sigma_{\mathrm{P}} \nabla \varphi_{\mathrm{P}} \cdot \boldsymbol{n}_{\mathrm{P}}
$$

where $\varphi_{\mathrm{wP}}$ and $\varphi_{\mathrm{wN}}$ denote the face potential in the owner and neighbor cell and $\Delta \varphi$ the jump between both; $\sigma_{\mathrm{P}}$ and $\sigma_{\mathrm{N}}$ denote (cell centered) conductivities, $\boldsymbol{n}_{\mathrm{P}}$ and $\boldsymbol{n}_{\mathrm{N}}$ the face normal vectors and $\varphi_{\mathrm{N}}$ and $\varphi_{\mathrm{P}}$ the cell values of the potential. Combining both above conditions leads to the face potential in the owner cell as

$$
\varphi_{\mathrm{wP}}=f \cdot\left(\varphi_{\mathrm{N}}-\Delta \varphi\right)+(1-f) \cdot \varphi_{\mathrm{P}}
$$

with

$$
f=\frac{\delta_{\mathrm{P}} \cdot \sigma_{\mathrm{N}}}{\delta_{\mathrm{N}} \sigma_{\mathrm{P}}+\delta_{\mathrm{P}} \sigma_{\mathrm{N}}} .
$$

Here, $\delta_{\mathrm{P}}$ and $\delta_{\mathrm{N}}$ denote the distance between face and cell center for the owner and neighbor cell, respectively.

\section{Validation}

We use a simple analytical test case to validate our model. A 1-dimensional bar is made of two materials of different conductivity, and an artificial potential jump of $1 \mathrm{~V}$ is applied between both. Further, a constant potential is applied at both ends of the bar, such that a potential profile as illustrated in fig.2 develops. The following parameters are used:

$$
\begin{aligned}
a & =0 \mathrm{~V}, \quad b=5 \mathrm{~V}, \quad c=1 \mathrm{~V}, \\
x_{0} & =0 \mathrm{~m}, \quad x_{1}=-2 \mathrm{~m}, \quad x_{2}=2 \mathrm{~m}, \\
\sigma_{1} & =10 \mathrm{~S} / \mathrm{m}, \quad \sigma_{2}=1 \mathrm{~S} / \mathrm{m} .
\end{aligned}
$$


The analytical solution predicts the potential profile as two lines:

$$
\begin{aligned}
& \varphi_{1}(x)=\alpha x+\beta, \\
& \varphi_{2}(x)=\gamma x+\varepsilon .
\end{aligned}
$$

with

$$
\begin{aligned}
& \alpha=\sigma_{2} \frac{b-c-a}{\sigma_{2}\left(h_{0}-h_{1}\right)-\sigma_{1}\left(h_{0}-h_{2}\right)}, \\
& \beta=a-\alpha h_{1}, \quad \gamma=\alpha \frac{\sigma_{1}}{\sigma_{2}}, \quad \varepsilon=b-\gamma h_{2} .
\end{aligned}
$$

The simulated electric potential in fig. 2 shows the jump as expected, and fits perfectly to the analytical solution. Moreover, the current density (not shown here) is continuous over the interface, and fits again to the analytical solution.

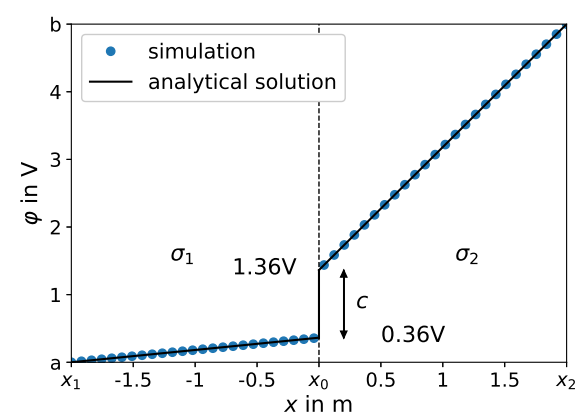

Figure 2: Electric potential distribution along a line for the one-dimensional test case. The potential at the boundaries (a, b) as well as the voltage jump (c) and the two conductivities $\left(\sigma_{1}, \sigma_{2}\right)$ are provided as initial conditions.

\section{Application to a Li || $\mathrm{Bi}$ liquid metal battery}

Liquid metal batteries are discussed as cheap stationary energy storage for fluctuating renewable energies $[59,60]$. We simulate a Li || Bi liquid metal battery [61, 62] in 3D (for a 1D model, see [63]) - and compare with measured data. The concentration cell consists of a Bi positive electrode (0.1 mol), a LiF-LiClLiI molten salt electrolyte and a Li negative electrode 64. The lithium is contained in a nickel spiral, and the vessel is made of tantalum to avoid as far 
as possible any reaction with the active materials and the electrolyte. Fig. 3 shows the setup, and tab.1 gives the material properties. The cell is heated from below such that the temperature at the bismuth-electrolyte interface stays at approximately $460^{\circ} \mathrm{C}$. Ultra-dry LiI is vacuum dried for $12 \mathrm{~h}$ while being heated step wise from 100 to $300^{\circ} \mathrm{C}$. $\mathrm{LiF}-\mathrm{LiCl}$ is vacuum dried for $12 \mathrm{~h}$ at $500^{\circ}$. Finally, LiF-LiCl-LiI is mixed in eutectic composition [65] and filtered through a quartz frit. The cell is cycled at $1 \mathrm{~A}$ with a cycle length of $10 \mathrm{~min}$.

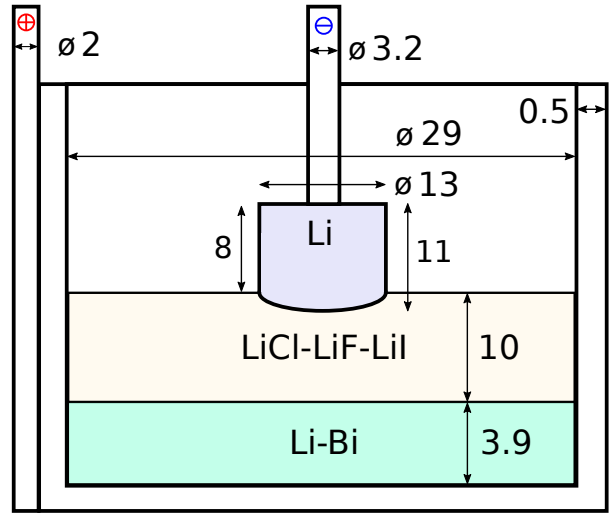

Figure 3: Experimental Li||Bi cell. The vessel is made of tantalum, the wires of copper. The lithium metal is contained in a spiral made of nickel. The Li-Bi layer thickness corresponds to a Li molar fraction of 0.236 .

The numerical model is simplified in three ways. Firstly, we insert a very thin gap artificially between electrolyte and vessel, because no current is allowed to flow there. Secondly, we model the negative electrode as it would consist of pure lithium. This is justified because the electric conductivities of nickel and lithium are very similar. Finally, we do not know the exact shape of the electrolyte-negative electrode interface - the exact immersion depth shown in fig. 3 is therefore an assumption. The numerical representation of the cell is shown in fig. 4a: 100 grid cells are used on the diameter of the FVM mesh, and the electrolyte-positive electrode interface is strongly refined. The potential is set to $0 \mathrm{~V}$ at the outer cable while a Neumann boundary condition corresponding to $1 \mathrm{~A}$ is applied at the negative contact. The open circuit potential at $460^{\circ} \mathrm{C}$ 
is fitted using measurement values [66] as

$$
E / \mathrm{V}=\frac{0.19}{x+0.41}+0.5
$$

for the interval of molar fraction $0.1<x<0.3$. The density of the Li-Bi alloy is computed using Vegard's law [67, 68.

Table 1: Material properties at $460^{\circ} \mathrm{C}[69+74]$.

\begin{tabular}{rrrrrrr}
\hline property & \multicolumn{1}{c}{$\mathrm{Li}$} & \multicolumn{1}{c}{$\mathrm{Bi}$} & salt & $\mathrm{Cu}$ & $\mathrm{Ta}$ & $\mathrm{Ni}$ \\
\hline$\rho$ in $\mathrm{kg} / \mathrm{m}^{3}$ & 490 & 9831 & 2690 & 2800 & & \\
$\sigma$ in $\mathrm{S} / \mathrm{m}$ & $2.7 \cdot 10^{6}$ & $7.2 \cdot 10^{5}$ & 271 & $58 \cdot 10^{6}$ & $2.9 \cdot 10^{6}$ & $3 \cdot 10^{6}$ \\
\hline
\end{tabular}

Fig.4 illustrates the electric potential field, and fig. $4 \mathrm{~d}$ the profile along the axis. We clearly see the potential jump corresponding to the open circuit voltage. The current density, as illustrated in fig.4 shows only an asymmetry at the bottom. Despite all current needs to flow to the lateral cable, the current distribution in the electrolyte is almost symmetric. Finally, fig. 4 f shows the 10 min discharge cycle of $1 \mathrm{~A}$, starting with a molar fraction of $\mathrm{Li}$ in $\mathrm{Bi}$ of $x=0.236$. Experiment and simulation fit very well. The corresponding Li molar fraction in Bi after 1, 5 and 10 minutes is shown in fig.4. As expected from the current distribution in fig. $4 \mathrm{~b}$, we find more $\mathrm{Li}$ in the center of the positive electrode. In reality, this could finally lead to the formation of intermetallic phases there.

\section{Summary and outlook}

We have discussed different approaches for modeling the cell voltage, as well as the macroscopic potential and current distribution in electrochemical cells. Special attention was paid to potential theory and the coupling of equations and different conductors. Thereafter, we have developed a three dimensional model for internal potential jumps in electrochemical cells, and implemented it into the finite volume method. An internal boundary condition, included into the 


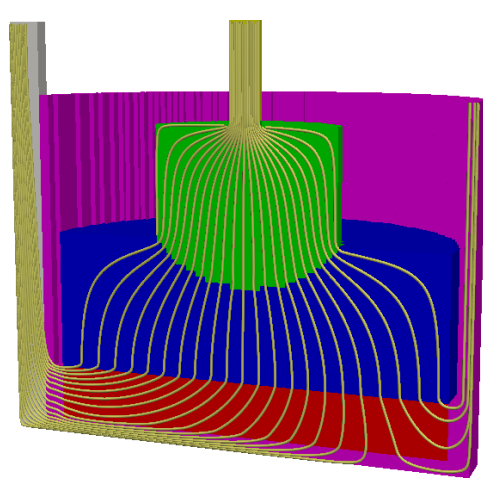

(a)

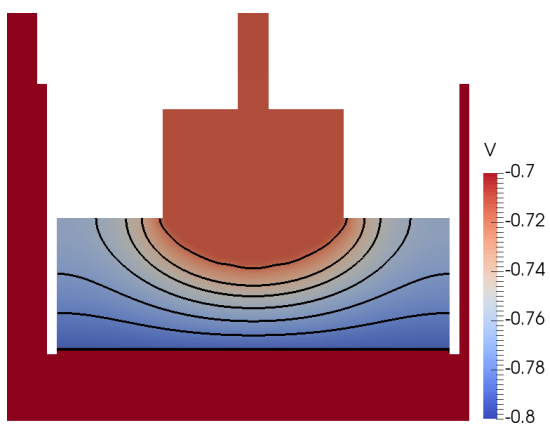

(c)

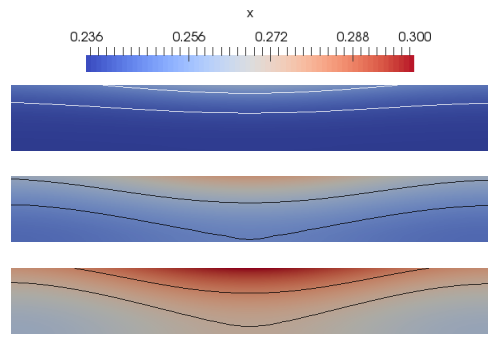

(e)

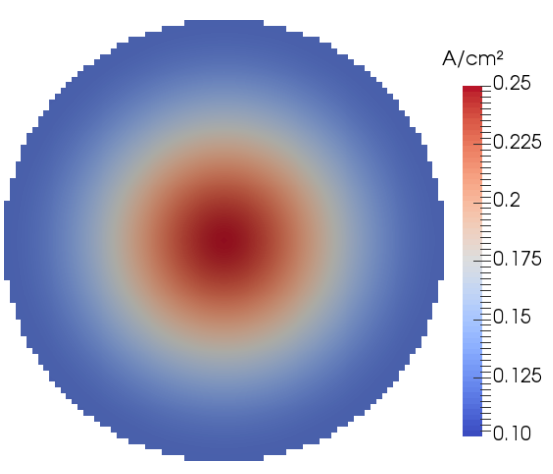

(b)

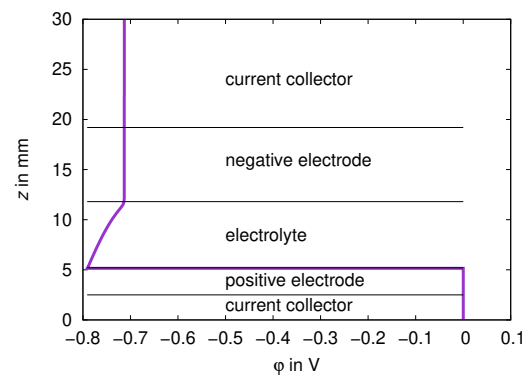

(d)

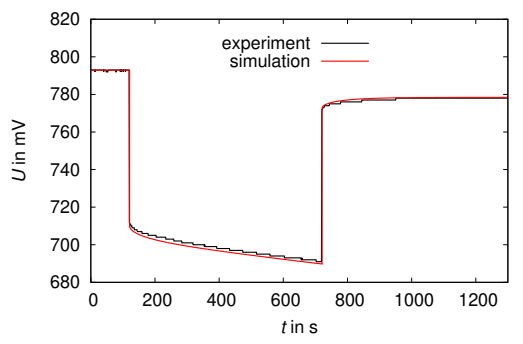

(f)

Figure 4: Grid with current distribution (a), current distribution at the electrolyte-positive electrode interface (b) and electric potential (c) in a Li||Bi liquid metal battery with a molar fraction of $\mathrm{Li}$ in $\mathrm{Bi}$ of $x=0.236$, as well as the vertical potential distribution along the axis of the cell (d). The discharge curve (f) matches fairly well between experiment and simulation; (e) shows the corresponding Li-molar fraction in the positive electrode after 1, 5 and 10 minutes. 
Laplace and gradient operator allows for arbitrary floating potential jumps at electrode-electrolyte interfaces. As it can compute the full cell potential in 3D at one single mesh, the model is highly efficient, robust and universal. It is fully parallelized and can work in galvano- and potentiostatic mode. After validating the model by a simple analytical formula we have illustrated its capabilities by simulating a Li ||Bi liquid metal battery and comparing with experimental data. We have correctly predicted the discharge cycle of the cell, and illustrated the current and potential distribution as well as the lithium concentration in bismuth.

Our discretization of the Laplace and gradient terms is quite universal: only a face field describing the jump needs to be provided. Our model can therefore be employed for modeling potential distributions in arbitrary electrochemical cells. Further, it has not escaped our notice that jumps can appear in electromagnetic fields, in temperature and potential fields due to contact resistance [75] or in concentrations fields at interfaces [29, as well. For all those applications, our model might potentially be useful.

In the future we plan to employ the developed model for studying the influence of mass transport on the cell potential of liquid metal batteries [76]. Specifically, we would like to extend the model by convection [18, 19, heat transfer [73, 77] and electrode kinetics.

\section{Acknowledgment}

This work was supported by the Deutsche Forschungsgemeinschaft (DFG, German Research Foundation) by award number 338560565 as well as a postdoc fellowship of the German Academic Exchange Service (DAAD). The computations were performed on a PC-Cluster at the Center for Information Services and High Performance Computing (ZIH) at TU Dresden and on the cluster Hemera at Helmholtz-Zentrum Dresden - Rossendorf. Fruitful discussions with S. Beale, V. Galindo, G. Mutschke and U. Reimer are gratefully acknowledged. Kashif Mushtaq is grateful to the Portuguese Foundation for Science and Tech- 
nology (FCT) for his PhD grant (reference: PD/BD/128041/2016) and support from the MIT Portugal Program.

\section{References}

[1] G. A. Prentice, A Survey of Numerical Methods and Solutions for Current Distribution Problems, J. Electrochem. Soc. 129 (1) (1982) 72 (1982). doi: $10.1149 / 1.2123795$

[2] J. O. Dukovic, Computation of current distribution in electrodeposition, a review, IBM J. Res. Dev. 34 (5) (1990) 693-705 (1990).

[3] A. Z. Weber, J. Newman, Modeling Transport in Polymer-Electrolyte Fuel Cells, Chem. Rev. 104 (10) (2004) 4679-4726 (Oct. 2004). doi:10.1021/ cr0207291.

[4] K. J. Vetter, Electrochemical Kinetics Theoretical Aspects, Elsevier Science, Burlington, 1967 (1967).

[5] P. Pierini, Current Distribution on a Disk Electrode for Redox Reactions, J. Electrochem. Soc. 123 (3) (1976) 366-369 (1976). doi:10.1149/1. 2132826 .

[6] J. Newman, K. E. Thomas-Alyea, Electrochemical Systems, John Wiley \& Sons, 2004 (2004).

[7] M. Ismail, D. Ingham, K. Hughes, L. Ma, M. Pourkashanian, An efficient mathematical model for air-breathing PEM fuel cells, Appl. Energy 135 (2014) 490-503 (Dec. 2014). doi:10.1016/j. apenergy.2014.08.113.

[8] S. B. Beale, H.-W. Choi, J. G. Pharoah, H. K. Roth, H. Jasak, D. H. Jeon, Open-source computational model of a solid oxide fuel cell, Comput. Phys. Commun. 200 (2016) 15-26 (Mar. 2016). doi:10.1016/j.cpc.2015.10. 007. 
[9] J. Deconinck, Calculation of Current Distribution and Electrode Shape Change by the Boundary Element Method, J. Electrochem. Soc. 132 (12) (1985) 2960-2965 (1985). doi:10.1149/1.2113701.

[10] M. García-Camprubí, Multiphysics Models for the Simulation of Solid Oxide Fuel Cells, Ph.D. thesis, University of Zaragoza, Zaragoza (2011).

[11] A. N. Colli, H. H. Girault, Compact and General Strategy for Solving Current and Potential Distribution in Electrochemical Cells Composed of Massive Monopolar and Bipolar Electrodes, J. Electrochem. Soc. 164 (11) (2017) E3465-E3472 (2017). doi:10.1149/2.0471711jes.

[12] J. S. Newman, C. W. Tobias, Theoretical Analysis of Current Distribution in Porous Electrodes, J. Electrochem. Soc. 109 (12) (1962) 1183-1191 (1962). doi:10.1149/1.2425269.

[13] J. Newman, W. Tiedemann, Porous-electrode theory with battery applications, AIChE J. 21 (1) (1975) 25-41 (Jan. 1975). doi:10.1002/aic. 690210103.

[14] M. Doyle, J. Newman, The use of mathematical modeling in the design of lithium/polymer battery systems, Electrochimica Acta 40 (1995) 21912196 (Oct. 1995). doi:10.1016/0013-4686(95)00162-8.

[15] T. F. Fuller, Simulation and Optimization of the Dual Lithium Ion Insertion Cell, J. Electrochem. Soc. 141 (1) (1994) 1-10 (1994). doi:10.1149/1. 2054684

[16] B. Paxton, Modeling of Nickel/Metal Hydride Batteries, J. Electrochem. Soc. 144 (11) (1997) 3818-3831 (1997). doi:10.1149/1.1838098.

[17] K. B. Hueso, M. Armand, T. Rojo, High temperature sodium batteries: Status, challenges and future trends, Energy Environ. Sci. 6 (3) (2013) 734-749 (2013). doi:10.1039/c3ee24086j. 
[18] R. Ashour, D. H. Kelley, A. Salas, M. Starace, N. Weber, T. Weier, Competing forces in liquid metal electrodes and batteries, J. Power Sources 378 (2018) 301-310 (2018). doi:10.1016/j.jpowsour.2017.12.042

[19] N. Weber, M. Nimtz, P. Personnettaz, A. Salas, T. Weier, Electromagnetically driven convection suitable for mass transfer enhancement in liquid metal batteries, Appl. Therm. Eng. 143 (2018) 293-301 (2018). doi:10.1016/j.applthermaleng.2018.07.067.

[20] P. Biesheuvel, M. van Soestbergen, M. Bazant, Imposed currents in galvanic cells, Electrochimica Acta 54 (21) (2009) 4857-4871 (Aug. 2009). doi: $10.1016 / \mathrm{j}$. electacta.2009.03.073

[21] W. A. van Schalkwijk, B. Scrosati (Eds.), Advances in Lithium-Ion Batteries, Kluwer Academic/Plenum Publishers, New York, NY, 2002 (2002).

[22] C. Y. Wang, W. B. Gu, Micro-Macroscopic Coupled Modeling of Batteries and Fuel Cells, J. Electrochem. Soc. 145 (1998) 3407-3417 (1998).

[23] A. J. Bard, L. R. Faulkner, Electrochemical Methods: Fundamentals and Applications, 2nd Edition, Wiley, New York, 2001 (2001).

[24] J. Lück, A. Latz, Theory of reactions at electrified interfaces, Phys. Chem. Chem. Phys. 18 (27) (2016) 17799-17804 (2016). doi:10.1039/ C6CP02681H.

[25] J. Klingert, S. Lynn, C. Tobias, Evaluation of current distribution in electrode systems by high-speed digital computers, Electrochimica Acta 9 (3) (1964) 297-311 (Mar. 1964). doi:10.1016/0013-4686(64)80019-0.

[26] S. B. Beale, Use of Streamwise Periodic Boundary Conditions for Problems in Heat and Mass Transfer, J. Heat Transf. 129 (4) (2007) 601-605 (2007). doi:10.1115/1.2709976.

[27] K. A. Smith, C. D. Rahn, C.-Y. Wang, Control oriented 1D electrochemical model of lithium ion battery, Energy Convers. Manag. 48 (9) (2007) 25652578 (Sep. 2007). doi:10.1016/j.enconman.2007.03.015. 
[28] J. Feinauer, S. Hein, S. Rave, S. Schmidt, D. Westhoff, J. Zausch, O. Iliev, A. Latz, M. Ohlberger, V. Schmidt, MULTIBAT: Unified workflow for fast electrochemical 3D simulations of lithium-ion cells combining virtual stochastic microstructures, electrochemical degradation models and model order reduction, J. Comput. Sci. (Mar. 2018). doi:10.1016/j . jocs. 2018. 03.006

[29] B. Kaoui, A simple algorithm to implement jump boundary conditions within the lattice Boltzmann method, arXiv:1811.01899 (Nov. 2018). arXiv:1811.01899.

[30] W. B. Gu, Numerical Modeling of Coupled Electrochemical and Transport Processes in Lead-Acid Batteries, J. Electrochem. Soc. 144 (6) (1997) 20532061 (1997). doi:10.1149/1.1837741.

[31] J. Euler, W. Nonnemacher, Stromverteilung in porösen Elektroden, Electrochimica Acta 2 (1960) 268-286 (1960).

[32] J. D. Milshtein, K. M. Tenny, J. L. Barton, J. Drake, R. M. Darling, F. R. Brushett, Quantifying Mass Transfer Rates in Redox Flow Batteries, J. Electrochem. Soc. 164 (11) (2017) E3265-E3275 (2017). doi:10.1149/2. 0201711jes.

[33] J. Newman, Engineering design of electrochemical systems, Ind. Eng. Chem. 60 (4) (1968) 12-27 (Apr. 1968). doi:10.1021/ie50700a005.

[34] J. Newman, Current Distribution and Mass Transfer in Electrochemical Systems, Symp. Electrochem. Process. 11 (1967) 50-54 (1967).

[35] R. E. White, On Newman's Numerical Technique for Solving Boundary Value Problems, Ind. Eng. Chem. Fundam. 17 (4) (1978) 367-369 (Nov. 1978). doi:10.1021/i160068a026.

[36] R. S. Varga, Matrix Iterative Analysis, 2nd Edition, no. 27 in Springer Series in Computational Mathematics, Springer Verlag, Berlin ; New York, $2000(2000)$. 
[37] M. C. Kimble, R. E. White, A five-point finite difference method for solving parabolic partial differential equations, Comput. Chem. Eng. 14 (1990) 921-924 (1990).

[38] T. Nguyen, R. White, A finite difference procedure for solving coupled, nonlinear elliptic partial differential equations, Comput. Chem. Eng. 11 (5) (1987) 543-546 (Oct. 1987). doi:10.1016/0098-1354(87)80029-7

[39] Z. Mao, A Finite-Difference Method for Pseudo-Two-Dimensional Boundary Value Problems, J. Electrochem. Soc. 141 (1) (1994) 151-156 (1994). doi:10.1149/1.2054675.

[40] H. Preisig, R. White, On the design of a simple solver for nonlinear twopoint boundary value problems, Comput. Chem. Eng. 14 (2) (1990) 179-196 (Feb. 1990). doi:10.1016/0098-1354(90)87077-3.

[41] G. G. Botte, V. R. Subramanian, R. E. White, Mathematical modeling of secondary lithium batteries, Electrochimica Acta 45 (15-16) (2000) 25952609 (May 2000). doi:10.1016/S0013-4686(00) 00340-6.

[42] S. B. Beale, U. Reimer, D. Froning, H. Jasak, M. Andersson, J. G. Pharoah, W. Lehnert, Stability Issues of Fuel Cell Models in the Activation and Concentration Regimes, J. Electrochem. Energy Convers. Storage 15 (4) (2018) 041008 (May 2018). doi:10.1115/1.4039858.

[43] T. Takayama, M. Yoneda, Modeling of thermal and electric behavior of stacked Lithium-ion cells in OpenFOAM, 6th OpenFOAM Workshop, Penn State University, USA (2011).

[44] A. Colli, J. Bisang, Current and potential distribution in electrochemical reactors with activated or resistive electrodes. A multiregion and open source approach, Electrochimica Acta 290 (2018) 676-685 (Nov. 2018). doi:10.1016/j.electacta.2018.09.121. 
[45] N. Weber, P. Beckstein, V. Galindo, M. Starace, T. Weier, Electro-vortex flow simulation using coupled meshes, Comput. Fluids 168 (2018) 101-109 (2018). doi:10.1016/j.compfluid.2018.03.047.

[46] R. E. White, Electrochemical Cell Design, Springer US, Boston, MA, 1984 (1984).

[47] E. C. Dimpault-Darcy, Secondary Current Distributions Using TOPAZ2D and Linear Kinetics, J. Electrochem. Soc. 135 (3) (1988) 656-658 (1988). doi:10.1149/1.2095692.

[48] D. Fan, Modification of Newman's BAND(J) Subroutine to Multi-Region Systems Containing Interior Boundaries: MBAND, J. Electrochem. Soc. 138 (6) (1991) 1688-1691 (1991). doi:10.1149/1.2085854.

[49] C. Ziegler, A. Schmitz, M. Tranitz, E. Fontes, J. O. Schumacher, Modeling Planar and Self-Breathing Fuel Cells for Use in Electronic Devices, J. Electrochem. Soc. 151 (12) (2004) A2028-A2041 (2004). doi: $10.1149 / 1.1810451$

[50] H. Ju, C.-Y. Wang, Experimental Validation of a PEM Fuel Cell Model by Current Distribution Data, J. Electrochem. Soc. 151 (11) (2004) A1954A1960 (2004). doi:10.1149/1.1805523

[51] E. Karimi-Sibaki, A. Kharicha, M. Wu, A. Ludwig, J. Bohacek, Contribution of an Electro-Vortex Flow to Primary, Secondary, and Tertiary Electric Current Distribution in an Electrolyte, J. Electrochem. Soc. 165 (11) (2018) E604-E615 (2018). doi:10.1149/2.1201811jes.

[52] H. G. Weller, G. Tabor, H. Jasak, C. Fureby, A tensorial approach to computational continuum mechanics using object-oriented techniques, Comput. Phys. 12 (6) (1998) 620-631 (1998).

[53] C. E. Vallet, Composition Gradients in Electrolyzed LiCl-KCl Eutectic Melts, J. Electrochem. Soc. 130 (12) (1983) 2366-2370 (1983). doi:10. 1149/1.2119588. 
[54] J. H. Ferziger, M. Perić, Computational Methods for Fluid Dynamics, Springer, 1996 (1996).

[55] H. Jasak, Error Analysis and Estimation for the Finite Volume Method with Applications to Fluid Flows, Ph.D. thesis, Imperial College of Science, Technology and Medicine (1996).

[56] S. Hill, D. Deising, T. Acher, H. Klein, D. Bothe, H. Marschall, Boundedness-Preserving Implicit Correction of Mesh-Induced Errors for VoF Based Heat and Mass Transfer, J. Comput. Phys. 352 (2018) 285-300 (Jan. 2018). doi:10.1016/j.jcp.2017.09.027.

[57] N. Van der Westhuizen, Gradient calculations of non-orthogonal meshes in the finite volume method, PhD Thesis, North-West University (2013).

[58] I. Demirdžić, On the Discretization of the Diffusion Term in Finite-Volume Continuum Mechanics, Numer. Heat Transf. Part B Fundam. 68 (1) (2015) 1-10 (Jul. 2015). doi:10.1080/10407790.2014.985992.

[59] H. Kim, D. A. Boysen, J. M. Newhouse, B. L. Spatocco, B. Chung, P. J. Burke, D. J. Bradwell, K. Jiang, A. A. Tomaszowska, K. Wang, W. Wei, L. A. Ortiz, S. A. Barriga, S. M. Poizeau, D. R. Sadoway, Liquid Metal Batteries: Past, Present, and Future, Chem. Rev. 113 (3) (2013) 2075-2099 (Mar. 2013). doi:10.1021/cr300205k.

[60] K. Wang, K. Jiang, B. Chung, T. Ouchi, P. J. Burke, D. A. Boysen, D. J. Bradwell, H. Kim, U. Muecke, D. R. Sadoway, Lithium-antimony-lead liquid metal battery for grid-level energy storage, Nature 514 (7522) (2014) 348-350 (Sep. 2014). doi:10.1038/nature13700.

[61] M. S. Foster, S. E. Wood, C. E. Crouthamel, Thermodynamics of Binary Alloys. I. The Lithium-Bismuth System, Inorg. Chem. 3 (10) (1964) 14281431 (1964).

[62] X. Ning, S. Phadke, B. Chung, H. Yin, P. Burke, D. R. Sadoway, Selfhealing Li-Bi liquid metal battery for grid-scale energy storage, J. Power 
Sources 275 (2015) 370-376 (Feb. 2015). doi:10.1016/j · jpowsour.2014. 10.173

[63] J. M. Newhouse, Modeling the Operating Voltage of Liquid Metal Battery Cells, Ph.D. thesis, Massachusetts Institute of Technology (2014).

[64] T. Weier, A. Bund, W. El-Mofid, G. M. Horstmann, C.-C. Lalau, S. Landgraf, M. Nimtz, M. Starace, F. Stefani, N. Weber, Liquid metal batteries - materials selection and fluid dynamics, IOP Conf. Ser. Mater. Sci. Eng. 228 (2017) 012013 (2017). doi:10.1088/1757-899X/228/1/012013.

[65] P. Masset, A. Henry, J.-Y. Poinso, J.-C. Poignet, Ionic conductivity measurements of molten iodide-based electrolytes, J. Power Sources 160 (1) (2006) 752-757 (Sep. 2006). doi:10.1016/j.jpowsour.2006.01.014.

[66] W. Gasior, Z. Moser, W. Zakulski, Bi-Li System. Thermodynamic properties and the phase diagram calculations, Arch. Metall. 39 (4) (1994) 355-364 (1994).

[67] L. Vegard, Die Konstitution der Mischkristalle und die Raumfüllung der Atome, Z. Für Phys. 5 (1921) 17-26 (1921).

[68] C. Fazio, Handbook on Lead-bismuth Eutectic Alloy and Lead Properties, Materials Compatibility, Thermal-hydraulics and Technologies, Tech. Rep. 7268, Nuclear Energy Agency (2015).

[69] S. J. Zinkle, Summary of Physical Properties for Lithium, Pb-17Li, and $(\mathrm{LiF}) \mathrm{n} \bullet \mathrm{BeF} 2$ Coolants, in: APEX Study Meeting, Sandia National Laboratories, 1998 (1998).

[70] V. Sobolev, Database of Thermophysical Properties of Liquid Metal Coolants for GEN-IV, SCK CEN, 2010 (2010).

[71] P. Masset, R. A. Guidotti, Thermal activated (thermal) battery technology, J. Power Sources 164 (1) (2007) 397-414 (Jan. 2007). doi:10.1016/j. jpowsour.2006.10.080. 
[72] C. J. Smithells, W. F. Gale, T. C. Totemeier, Smithells Metals Reference Book, 8th Edition, Elsevier Butterworth-Heinemann, Amsterdam ; Boston, 2004 (2004).

[73] P. Personnettaz, P. Beckstein, S. Landgraf, T. Köllner, M. Nimtz, N. Weber, $\mathrm{T}$. Weier, Thermally driven convection in Li $\|$ Bi liquid metal batteries, J. Power Sources 401 (2018) 362-374 (2018). doi:10.1016/j.jpowsour. 2018.08 .069

[74] D. R. Lide (Ed.), CRC Handbook of Chemistry and Physics, CRC Press, $2005(2005)$.

[75] Y. Wang, M. Ouyang, Three-dimensional heat and mass transfer analysis in an air-breathing proton exchange membrane fuel cell, J. Power Sources 164 (2) (2007) 721-729 (Feb. 2007). doi:10.1016/j.jpowsour.2006.11. 056.

[76] D. H. Kelley, T. Weier, Fluid mechanics of liquid metal batteries, Appl. Mech. Rev. 70 (2) (2018) 020801 (2018). doi:10.1115/1.4038699.

[77] Y. Shen, O. Zikanov, Thermal convection in a liquid metal battery, Theor. Comput. Fluid Dyn. 30 (4) (2016) 275-294 (2016). doi:10.1007/ s00162-015-0378-1. 